\title{
Effect of growth time on structural, morphological and electrical properties of tungsten oxide nanowire
}

\author{
S. A. A. Terohid ${ }^{1}$ · S. Heidari ${ }^{2}$ - A. Jafari ${ }^{1}$ - S. Asgary ${ }^{3}$
}

Received: 1 April 2018 / Accepted: 17 June 2018 / Published online: 27 July 2018

(c) The Author(s) 2018

\begin{abstract}
In this work, tungsten oxide nanowires were grown directly on a tungsten substrate in $800{ }^{\circ} \mathrm{C}$ using thermal evaporation method in a horizontal tube furnace. The effect of growth time on structural, morphological, elemental composition and electrical properties of the tungsten oxide nanostructures was investigated using X-ray diffraction (XRD), field emission scanning electron microscopy (FESEM), X-ray photoelectron spectroscopy (XPS) technique and KIETHLEY 2361 system. Our experimental results show that tungsten oxide nanowires crystalline phase, electrical conductance and density on the substrate surface depend on the growth time. The XRD results showed that tungsten oxide nanowires are synthesized with a cubic $\mathrm{WO}_{3}$ structure. Moreover, the lattice strain, grain size and dislocation density were obtained in three different growth time. It is noteworthy that by increasing the growth time the crystallinity increases. The FESEM images at different growth times showed that the nanowires on grains begin to germinate when the growth time increases to $6 \mathrm{~h}$. At this time, nanowire structures with $1 \mu \mathrm{m}$ in diameter and $40 \mu \mathrm{m}$ in length were formed and continued to grow which caused a change in the morphology. In addition, the XPS results showed that the sample that has grown at longer growth time exhibit two asymmetric $\mathrm{W} 4 d 5 / 2$ and $\mathrm{W} 4 d 3 / 2$ peak at 252 and $263 \mathrm{eV}$ which can be assigned to $\mathrm{W} 5+$ and $\mathrm{W} 4+$ species, respectively. Moreover, the linear I-V curves are obtained and it is found that by increasing the growth time, the conductivity of the sample increases due to increasing number of wires on the sample surface. Finally, the conditions and mechanism of $\mathrm{WO}_{3}$ nanowire growth are discussed. The results can be used to guide a better understanding about the growth behavior of $\mathrm{WO}_{3}$ nanowires and can contribute towards the development of novel nanodevices.
\end{abstract}

\section{Introduction}

Semiconductor oxides are very extraordinary substance in material science, industry and technology. Semiconductor oxides nanostructures have attracted much attention due to their potential in nanoelectronics and optoelectronics [1-5]. Among semiconductor oxides, tungsten oxide with various oxidation states of $\mathrm{WO}_{3-\mathrm{x}}$ has been recognized as one of the most impressive candidate compared with other electrochromic materials due to its fast optical response time. High coloration tungsten oxide nanowires

S. A. A. Terohid

terohid.saa@gmail.com

1 Department of Physics, Hamedan Branch, Islamic Azad University, Hamedan, Iran

2 Department of Mechanical Engineering, Amirkabir University of Technology, Tehran, Iran

3 Department of Physics, West Tehran Branch, Islamic Azad University, Tehran, Iran have attracted lots of attention, due to their potential in $\mathrm{pH}$ sensing, water splitting, gas sensors, n-type semiconductor, photocatalytic and field emission [6-9]. Hence tungsten oxide nanowires (NWs) have been synthesized by numerous synthetic approaches such as thermal evaporation, chemical vapor deposition (CVD), solvothermal route and electro spinning method for their electric or electrochemical applications [9-12]. Liu et al. [13] reported on the preparation of tungsten oxide nanowires through a vapor-solid growth process by heating a tungsten wire partially wrapped with boron oxide at $1200{ }^{\circ} \mathrm{C}$. In the works by Klinke et al. [14] and Yu et al. [15], related to the formation of $\mathrm{W}$ oxide NWs, a possible driving force for NWs growth is suggested to be the formation of a stressed state at the interface between the surface oxide and bulk metal. Gang $\mathrm{Gu}$ et al. investigated the formation of isolated tungsten oxide nanowires directly grown on tungsten tips and plates [16]. Moreover, Shim et al. reported electrochromic properties of polycrystalline $\mathrm{WO}_{3}$ nanowire electrodes fabricated on an indium tin oxide coated substrate 
by electrospinning method [17]. Among the mentioned methods, the direct growth of NWs has attracted much attention due to its simplicity, cheapness and samples high degree of crystallinity. However, most of the formed $\mathrm{WO}_{3-\mathrm{x}} \mathrm{NWs}$ are randomly dispersed, lacking substrate support with disordered distribution while for most applications, it is an important requisite to have the control on the orientation and position of $\mathrm{WO}_{3-\mathrm{x}} \mathrm{NWs}$ on proper substrates. Based on Dastan et al. results, it has been shown that the methods and reactant materials for producing a nanostructures (such as $\mathrm{TiO}_{2}$ nanoparticles) influences its morphological, structural, optical, molecular and thermal properties [4, 5]. Therefore, many researchers have prepared vertically aligned tungsten oxide nanostructures, this is because the vertically aligned nanostructures increase the path-length of the light through material for efficient light absorption with scattering, and while maintaining the path lengths reasonably short for the photo-generated charges to travel $[13,18,19]$. In this study, we present experimental results on the formation of tungsten oxide nanowire, which clearly demonstrate the effect of growth time on its morphology, structure, elemental composition and electrical properties using X-ray diffraction (XRD), field emission scanning electron microscopy (FESEM), X-ray photoelectron spectroscopy and KIETHLEY 2361 system. This paper is organized as follows: after the introduction, the experimental setup is explained in Sect. 2. The results and discussion are presented in Sects. 3 and 4 is devoted to the conclusion.

\section{Experimental detail}

\subsection{Fabrication of tungsten oxide nanowire}

A tungsten plate $(99.99 \%$, diameter $=25 \mathrm{~mm}$, thickness $=5.0 \mathrm{~mm}$ ) of about $1 \mathrm{~cm} \times 1 \mathrm{~cm}$ was used as the starting material and substrate. The tungsten plate was cleaned by ethanol (from Merck Germany Co.) under an ultrasonic bath for $5 \mathrm{~min}$, followed by being rinsed with de-ionized water. The tungsten plate was placed into a horizontal quartz tube (Yaran Behgozin Parsa Atmosphere Controlled Tube Furnace, YTF $1250-30 \times 8$ ) for evaporation and oxidation by the air. To control the growth process, the air oven temperature was monitored continuously by placing a thermocouple in the vicinity of the tungsten plate during the annealing in $800{ }^{\circ} \mathrm{C}$. The samples were prepared at three growth times of 4,5 and $6 \mathrm{~h}$. After the tungsten plate was oxidized, the samples were slowly cooled down to room temperature in the air oven to avoid thermal shocks and to achieve crackfree nanowire samples. Afterward a black layer was formed on the surface of the tungsten plate.

\subsection{Characterization techniques}

Crystalline structures were analyzed using X-ray diffraction (XRD). The diffraction patterns were recorded on an STOE-XRD diffractometer with $\mathrm{Cu} \mathrm{K} \alpha$ radiation $(\lambda=1.54060 \AA)$, the scanning rate of $0.2 \mathrm{~s}$ at $2 \theta=20-50^{\circ}$ and step size of $0.02^{\circ}$. Surface morphology was characterized by field emission scanning electron microscopy (FESEM, Leo 440i). X-ray photoelectron spectroscopy technique (Thermo Scientific, K-Alpha) was used to measure the elemental composition. Moreover to measure the electrical properties, a KIETHLEY 2361 system was used at room temperature.

\section{Result and discussion}

\subsection{XRD analysis}

The XRD method is used to analyze structure parameters such as microstrain, crystallite size and dislocation density. Figure 1 shows the X-ray diffraction patterns of tungsten oxide nanowire as a function of growth time with different morphologies. In the present work, all the XRD peaks could be indexed with a cubic $\mathrm{WO}_{3}$ structure. As the growth time increases, the number of sharp XRD peaks increases so XRD peak positions are identical because chemical compositions are identical among them. However, the intensity of each diffraction peak is different among them and at longer growth times $(t=6 \mathrm{~h})$; all the structures are well-crystallized with a mixture of $\mathrm{W}$ and $\mathrm{WO}_{3}$. Higher crystallinity of the tungsten oxide nanowire at longer growth time is attributed to ad-atoms that had enough time to forms a well-crystallized structure. In the other words, the formation of high intensity or sharp peaks is an indication for a higher degree of order, crystallization and arrangement. At shorter growth times $(t=4 \mathrm{~h})$ relatively weak diffraction peaks were observed (Table 1). This decrease in XRD peak intensity is an indication for a low degree of crystallinity. As growth time increased from 4 to 5 and to $6 \mathrm{~h}$ the crystallinity improved gradually and several new strong Bragg peaks were appeared forming a polycrystalline structure (listed in Tables 2, 3).

According to Fig. 1a, the A, B and C diffraction peaks at $2 \theta$ values of $25.84^{\circ}, 38.64^{\circ}$ and $44.92^{\circ}$ could be indexed as (101), (112) and (122) planes of the $\mathrm{WO}_{3}$ structure. The peak width of textured grains in the growth directions of (110) and (122) was very narrow, indicating a high degree of crystallinity $[20,21]$. The $\mathrm{C}$ and $\mathrm{G}$ peak intensities gradually increased to their maximum and the C peak shifted slightly to a lower angle position as the 

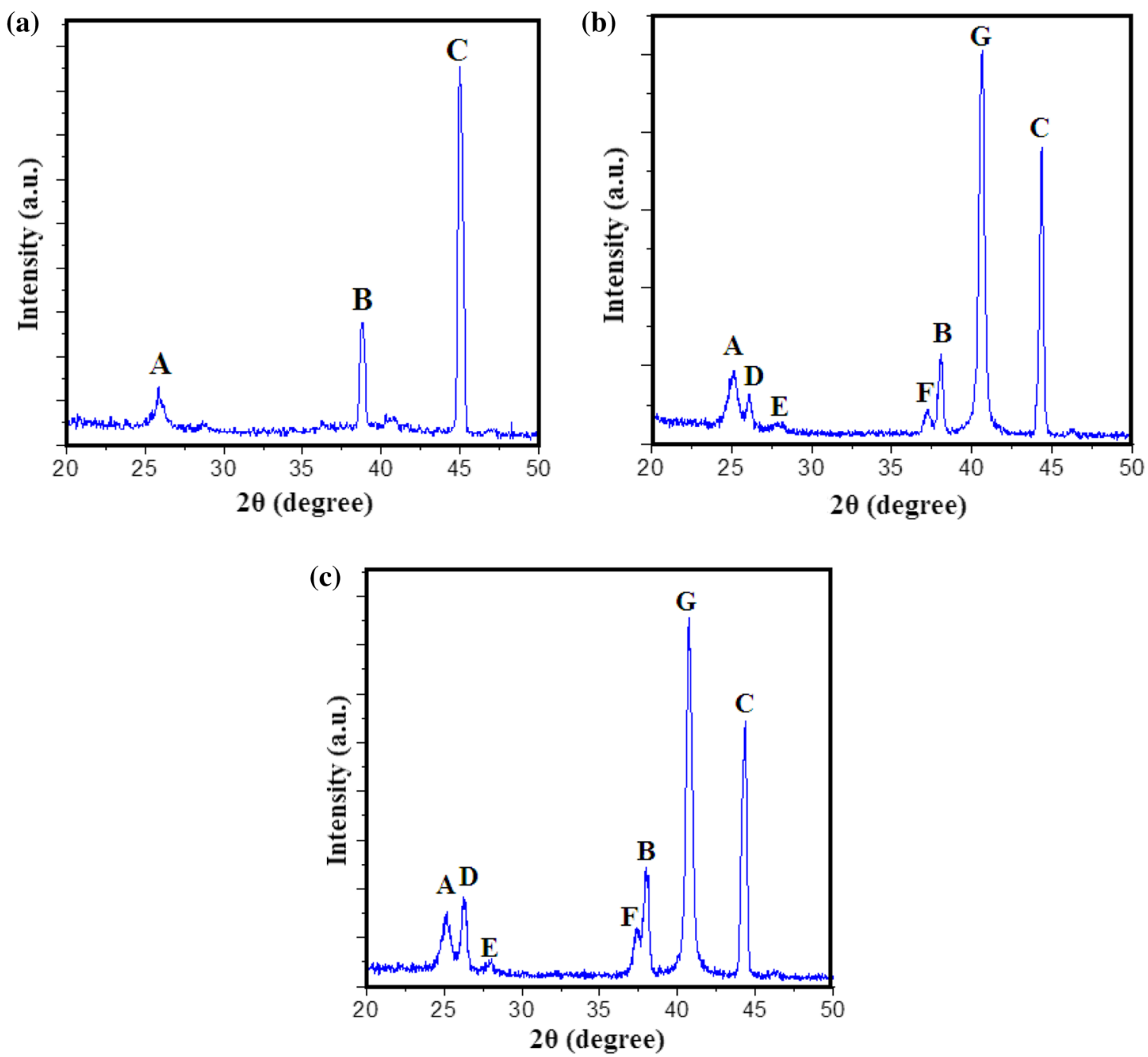

Fig. 1 XRD spectra of WO3 nanowire at different growth times: a 4, b 5 and $\mathbf{c} 6 \mathrm{~h}$

Table 1 Identification of XRD peaks in Fig. 1a

\begin{tabular}{lllllllll}
\hline Peak density & $2 \theta$ (degree) & FWHM & $\mathrm{d}(\AA)$ & hkl & Intensity $(\%)$ & $\begin{array}{l}\text { Crystallite } \\
\text { size }(\mathrm{nm})\end{array}$ & $\begin{array}{l}\text { Lattice } \\
\text { strain } \\
\left(10^{-3}\right)\end{array}$ & Dislocation \\
\hline A & 25.85 & 0.39 & 3.44 & 101 & 10.35 & 28 & 7.2 & 0.0012 \\
B & 38.64 & 0.19 & 3.18 & 112 & 26.76 & 70 & 2.3 & 0.0002 \\
C & 44.92 & 0.13 & 2.41 & 122 & 100.00 & 68 & 1.3 & 0.0002 \\
\hline
\end{tabular}

growth time increased, indicating that the lattice parameter increased with the increase in the growth time [20]. The peak at $40.70^{\circ}$ contributed to $\mathrm{W}(110)$ plane.

$\mathrm{XRD}$ analysis is also employed to identify details about phase compositions, crystal preferred orientations and crystal defect concentrations [21]. At shorter growth times the dominant orientation of $\mathrm{WO}_{3}$ nanowires is the (122) plane. At longer times a preferred growth along the (122) and (101) directions forms showing a light textured growth along these orientations. The ad-atoms accumulate in a way to occupy the minimum energy position by surface diffusion. Although anisotropy in growth is an inherent material property, deposition rate and surface of top layers influence the growth rate in particular planes [22]. When the growth time increased, the crystal growth direction of the surface became disordered, and more surface area was exposed to air. 
Table 2 Identification of XRD peaks in Fig. 1b

\begin{tabular}{lllllclcc}
\hline Peak density & $2 \theta$ (degree) & FWHM & $\mathrm{d}(\AA)$ & hkl & Intensity $(\%)$ & $\begin{array}{l}\text { Crystallite } \\
\text { size }(\mathrm{nm})\end{array}$ & $\begin{array}{l}\text { Lattice } \\
\text { strain } \\
\left(10^{-3}\right)\end{array}$ & Dislocation \\
\hline A & 25.16 & 0.27 & 3.53 & 101 & 15.65 & 35 & 5.8 & 0.0008 \\
D & 26.08 & 0.23 & 3.41 & 201 & 9.45 & 47 & 11.3 & 0.0004 \\
E & 27.99 & 0.62 & 3.18 & 230 & 1.44 & 14 & 10.5 & 0.0051 \\
F & 37.21 & 0.39 & 2.41 & 202 & 6.25 & 23 & 4.9 & 0.0018 \\
B & 38.15 & 0.25 & 2.35 & 112 & 9.58 & 46 & 3.0 & 0.0004 \\
G & 40.70 & 0.27 & 2.21 & 110 & 100.00 & 46 & 3.0 & 0.0004 \\
C & 44.38 & 0.21 & 2.03 & 122 & 75.88 & 46 & 2.1 & 0.0004 \\
\hline
\end{tabular}

Table 3 Identification of XRD peaks in Fig. 1c

\begin{tabular}{lllllllll}
\hline Peak density & 20(degree) & FWHM & $\mathrm{d}(\AA)$ & $\mathrm{hkl}$ & Intensity $(\%)$ & $\begin{array}{l}\text { Crystallite } \\
\text { size }(\mathrm{nm})\end{array}$ & $\begin{array}{l}\text { Lattice } \\
\text { strain } \\
\left(10^{-3}\right)\end{array}$ & Dislocation \\
\hline $\mathrm{A}$ & 25.16 & 0.62 & 3.53 & 101 & 14.42 & 14 & 5.8 & 0.0051 \\
$\mathrm{D}$ & 26.20 & 0.31 & 3.40 & 201 & 17.96 & 28 & 5.6 & 0.0012 \\
$\mathrm{E}$ & 27.94 & 0.47 & 3.19 & 230 & 2.92 & 20 & 8.0 & 0.0025 \\
$\mathrm{~F}$ & 37.20 & 0.23 & 2.40 & 202 & 12.34 & 46 & 2.9 & 0.0004 \\
$\mathrm{~B}$ & 38.10 & 0.35 & 2.36 & 112 & 25.73 & 28 & 4.3 & 0.0012 \\
$\mathrm{G}$ & 40.73 & 0.09 & 2.21 & 110 & 100.00 & 39 & 4.1 & 0.00005 \\
$\mathrm{C}$ & 44.37 & 0.16 & 2.03 & 122 & 69.04 & 70 & 6.6 & 0.0002 \\
\hline
\end{tabular}

The grain size was estimated using Scherrer's formula [23]:

$D=\frac{0.9 \lambda}{\beta \cos \theta}$,

where $\lambda$ is the wavelength of $\mathrm{Cu} \mathrm{K \alpha} \beta$ is the FWHM of the diffraction peak, $\theta$ is the peak position, and $D$ is the crystallite size. The lattice strain, grain size and dislocation density are calculated and given in Tables 1,2 and 3. From the results, we could find that the crystallite size for dominant crystallographic (122) plane first decreased and then increased with increasing growth time.

One of the characteristics of XRD signature is line broadening. The line broadening of X-ray peaks is related not only to crystallite size or changes in lattice structure but also to the function of internal strain ' $\varepsilon$ ' that is developed during the deposition process. The broadening due to strain is caused by the non-uniform displacements of the atoms with respect to their reference-lattice positions [24]. The deviation from the perfect lattice arrangement is calculated using Stokes-Wilson equation:

$\varepsilon=\beta / 4 \tan \theta$,

where ' $\beta$ ' is the FWHM of the peaks and ' $\theta$ ' is Braggs Angle [23].If the strains are large, their effect on the peakbroadening can be significant. Real crystals are not perfectly arranged as they have some lattice imperfection; such as stacking faults, dislocation, grain boundaries, etc. The lattice spacing $\left(\mathrm{d}_{122}\right)$ has decreased at longer growth times, which suggests a reduced internal strain as well as the formation of uniform grains. The internal strain has also increased at longer growth times which is attributed to an increase in lattice imperfection. The thermal gradient is one of the possible reasons for lattice imperfection. This behavior could refer to the reduction of the interstitial defects [25].

The dislocation density $(\delta)$ can be obtained using Williamson and Smallman relation [25].The higher grain size ' $D$ ' indicates the lower dislocation lines/volume with a better crystallinity.

$\delta=1 / D^{2}$,

where ' $D$ ' is the grain size calculated by Debye Scherrer's formula. In this formula, dislocations are assumed to be isotropically distributed in crystal and it lie along the grain boundary [26]. The dislocation density for dominant (122) plane has increased first and then decreased with increasing the growth time.

\subsection{X-ray photoelectron spectroscopy}

Differences between the initial and final states of an atom when a core level electron is excited by an X-ray is fundamental to X-ray photoelectron spectroscopy (XPS) as an analytical technique. High sensitivity surface analysis of 
XPS testing was utilized to identify the elemental composition, chemical state, oxidation state, and electronic state of the sample surface [27]. The increase in counts as a function of kinetic energy associates with the excitation of a core level electron appearing as an ensemble of peaks rather than a single peak. Such chemically shifted peaks offer information about the chemistry of the surface. The changes in the surface chemical states of three tungsten oxide nanowires grown at different times were monitored using XPS. Figures $(2,3,4,5,6)$ show the XPS of W4 $f$ and W5 $p$ core levels spectra for the samples prepared in different growth times which could be deconvoluted into two peaks corresponding
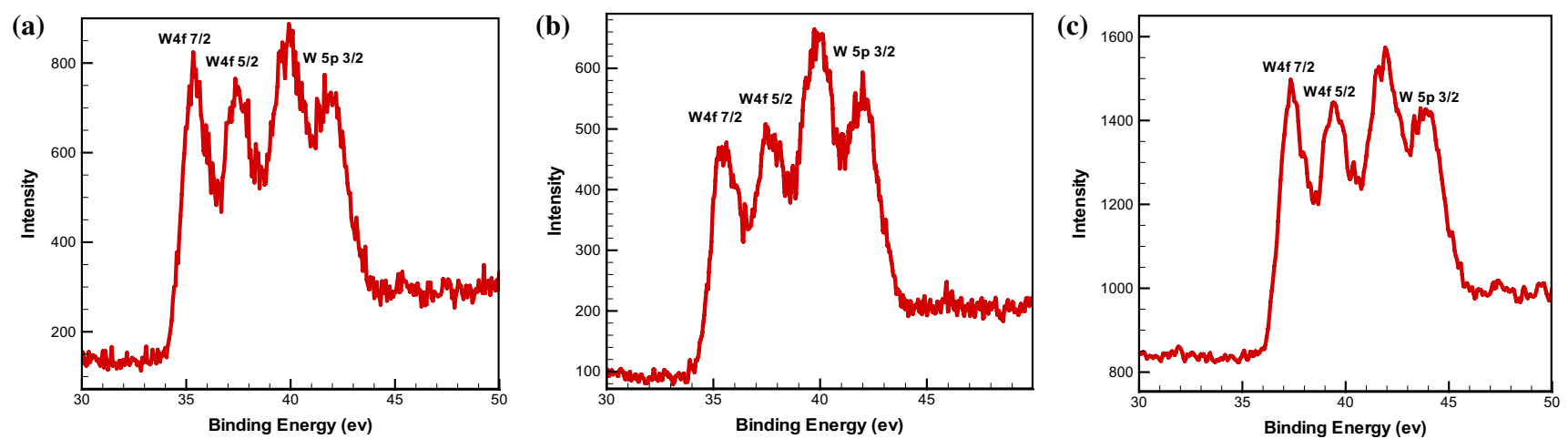

Fig. 2 W $4 f$ XPS and W5p spectra of samples that grown at different time a 4, b 5 and $\mathbf{c} 6 \mathrm{~h}$
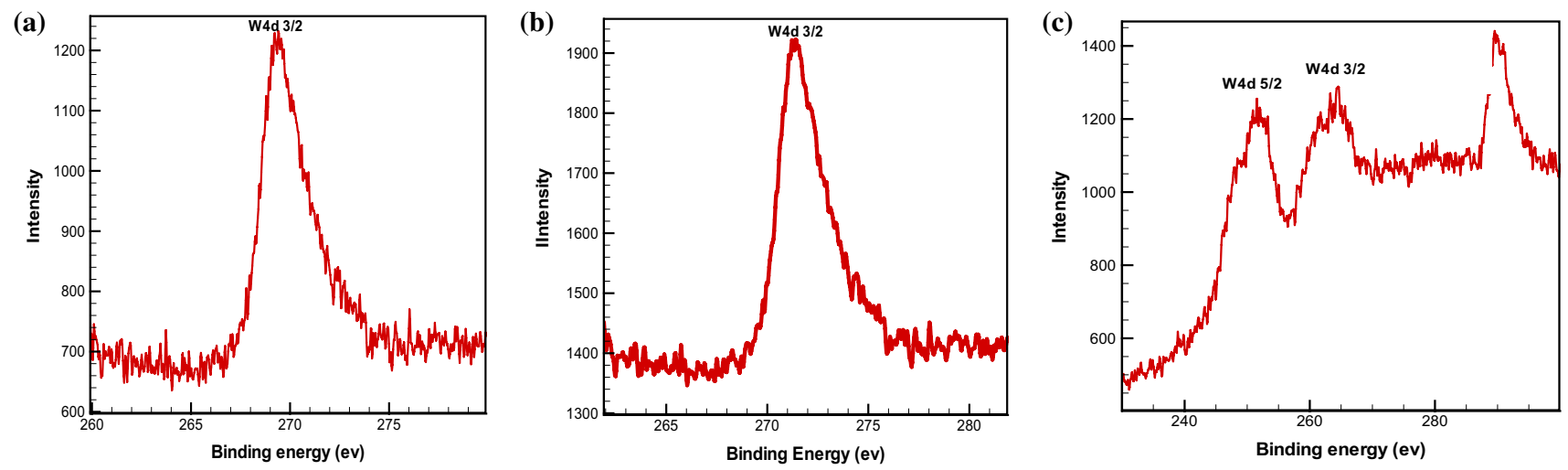

Fig. 3 W4d XPS spectra of samples that grown at different time a 4, b 5 and $\mathbf{c} 6 \mathrm{~h}$
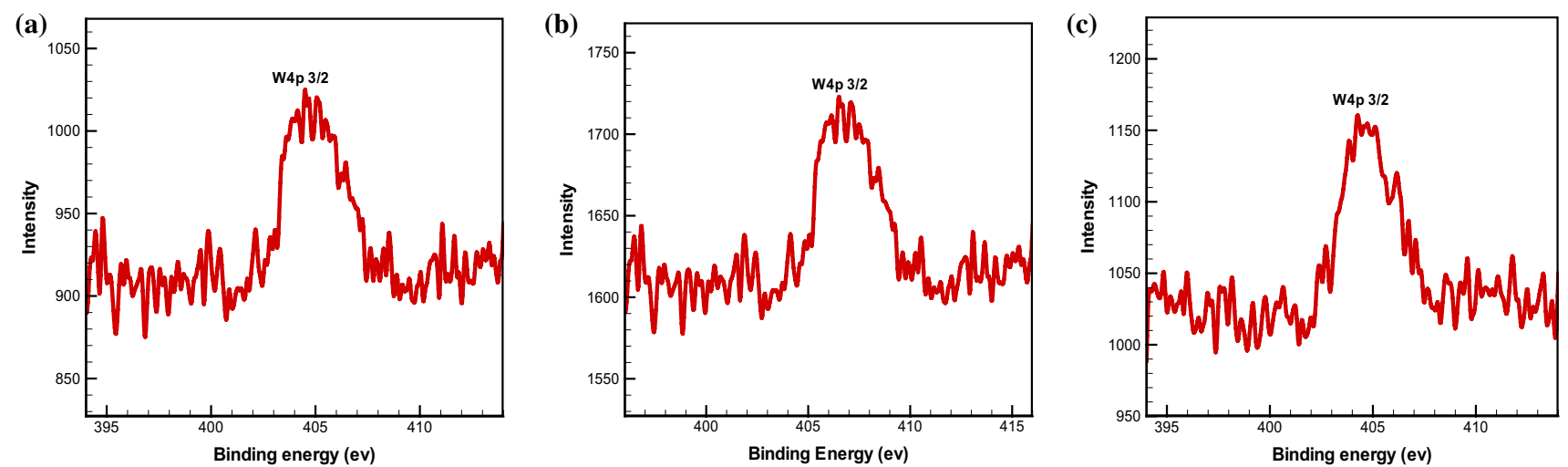

Fig. $4 \mathrm{~W} 4 p$ XPS spectra of samples that grown at different time a 4, b 5 and $\mathbf{c} 6 \mathrm{~h}$ 

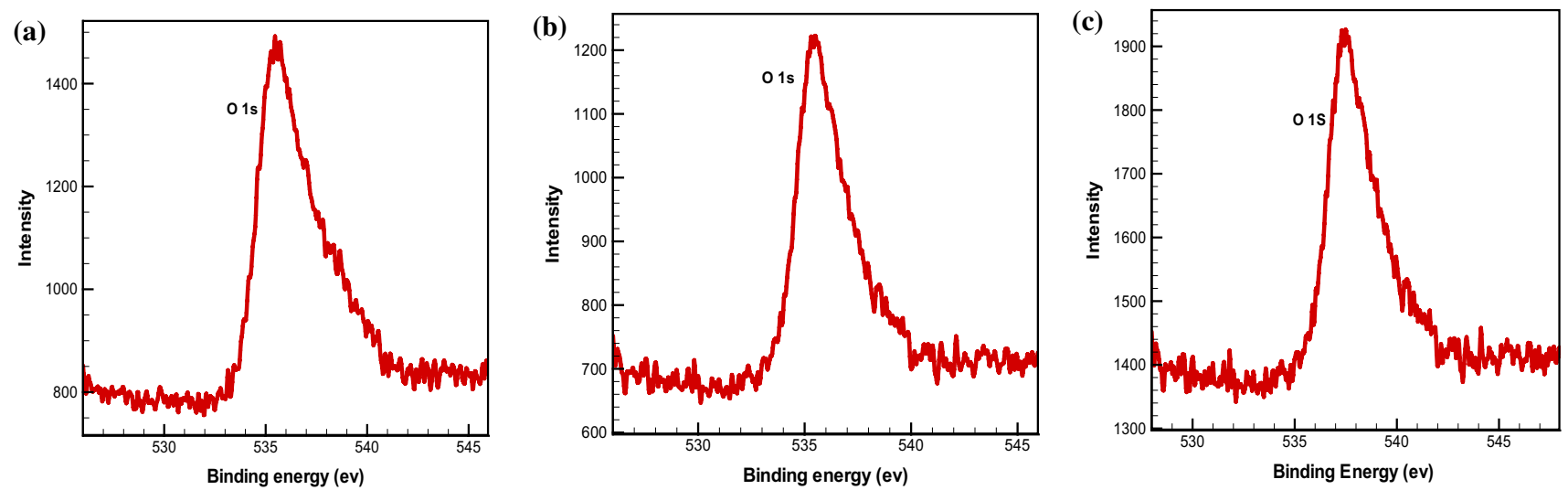

Fig. 5 O1s XPS spectra of samples that grown at different time a 4, b 5 and c 6 h
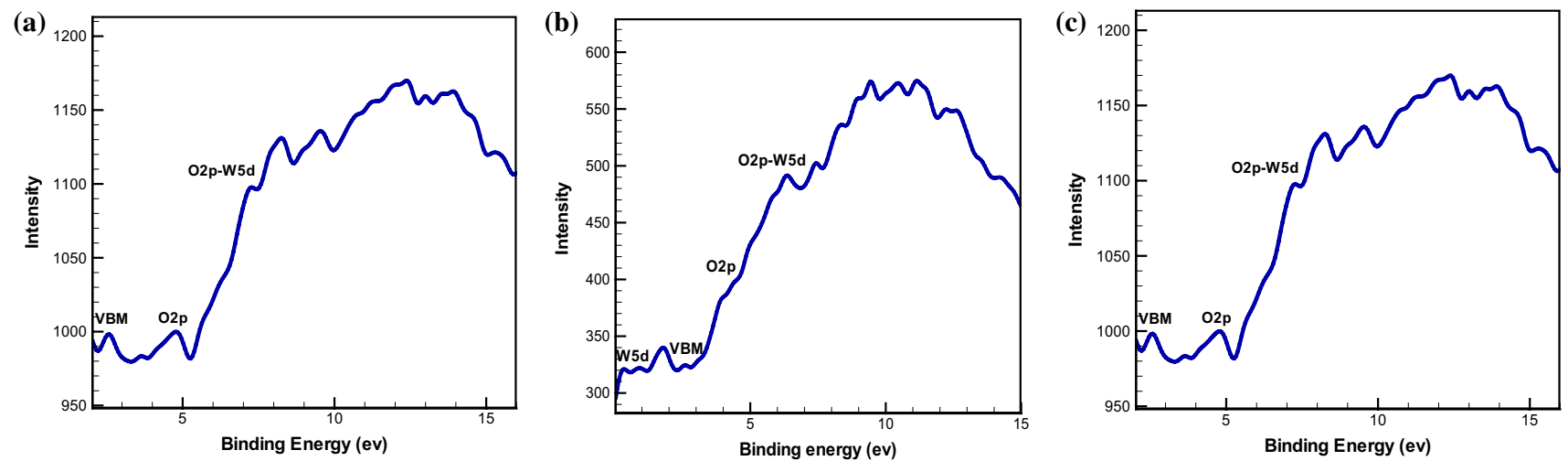

Fig. 6 Valence band spectra of samples that grown at different time a 4, b 5 and $\mathbf{c} 6 \mathrm{~h}$

to $\mathrm{W}^{6}$ state. The binding energy (BE) at the positions 35.5 and $37.6 \mathrm{eV}$ with peak separation of $2.1 \mathrm{eV}$ corresponds to the binding energy of W $4 f 7 / 2$ and $\mathrm{W} 4 f 5 / 2$ levels of tungsten atoms for $\mathrm{W}^{6+}$ state of oxide [28]. The positions of the two peaks for $\mathrm{W}^{5+}$ are located at lower binding energies, specifically at 34.9 and $37.1 \mathrm{eV}$. Even smaller binding energies, at 33.5 and $35.8 \mathrm{eV}$, are expected for $\mathrm{W}^{4+}$ [29].

The W $4 f$ core-level binding energy displays a rather strong dependency on the oxidation state of the $\mathrm{W}$ atom. Bonding to oxygen atoms shifts these two peaks to higher binding energies from the usual position of metallic tungsten $(31.4$ and $33.6 \mathrm{eV})$ to $36 \mathrm{eV}[29,30]$. It should be noted that given the rather high energies of the available $\mathrm{X}$-ray excitation $(1253.6 \mathrm{eV})$, the surface sensitivity for $\mathrm{W} 4 f$ photoelectrons is not very high [30].

The peak's positions for both samples that were grown at 4 and $5 \mathrm{~h}$ were not affected. However, all peaks in the sample which was grown in $6 \mathrm{~h}$ were shifted to higher BE compared to that other samples. It might be due to the excitation of electron-hole-pair states, namely, interactions of the Fermi-gas conduction electrons with holes caused by the absorption of X-rays by the core electrons [31].The intensity of W $4 f 7 / 2$ of $\mathrm{W} 4 f$ core level varied and drastically increased at $6 \mathrm{~h}$ of growth time. The peak at $41.7 \mathrm{eV}$ was assigned to $\mathrm{W} 5 p$. The $\mathrm{W} 5 p 3 / 2$ peak was in the higher BE side of $\mathrm{W} 4 f 5 / 2$. The $\mathrm{W} 5 p 3 / 2$ invariably accompanied with the $\mathrm{W} 4 f$ peak with nearly unchangeable relative peak position and intensity [32].

The $\mathrm{WO}_{3}$ sample that was grown at $6 \mathrm{~h}$ exhibited two asymmetric peaks at 252 and $263 \mathrm{eV}$ which are assigned to $\mathrm{W} 4 d 5 / 2$ and $\mathrm{W} 4 d 3 / 2$, respectively, which can be assigned to $\mathrm{W}^{5+}$ and $\mathrm{W}^{4+}$ species, respectively $\left(\mathrm{W}^{6+}\right.$ species $>\mathrm{W}^{5+}$ species $>\mathrm{W}^{4+}$ species $)$. All of the $\mathrm{W}$ surface atoms were in the highest oxidation state in the form of crystalline $\mathrm{WO}_{3}[32,33]$. The peak shape, however, remained slightly less symmetric. The most drastic changes could be found for these peaks. The line shapes and relative intensities of the $\mathrm{W} 4 d 5 / 3$ spectrum were indistinguishable from the spectrum of the $\mathrm{WO}_{3}$ nanowire that was grown at 4 and $5 \mathrm{~h}$. In addition, the peak's position has significantly shifted to a higher BE with a sharp edge compared to the others. 
The XPS analysis (Fig. 5) yielded the value for the bond energy of the O1s electrons. $(535.5 \mathrm{eV})$. The $\mathrm{O} 1 \mathrm{~s}$ spectrum of each sample contains a main contribution at the lowest binding energy by Gaussian distribution, originated from the $\mathrm{W}-\mathrm{O}$ bond. The peak's position for two samples was not affected. However, the peak in the sample (c) was significantly displaced compared to the others. The relative intensity of this peak is higher in the sample (b). The O1s spectrum showed a little shoulder at the right side at sample (a). This peak mainly displays a change in symmetry, becoming more symmetric with increasing growth time.

The two prominent features in the spectrum are readily identified. The energy bands within the valence band of WO3 have almost the entire characteristics of $\mathrm{W} 5 d$ or $\mathrm{O} 2 p$ orbital [33]. Covalent bonding interactions transformed $\mathrm{O} 2 p$ and W5 $d$ orbitals into the bonding and antibonding orbitals. The typical XPS valence band spectrum of the $\mathrm{WO}_{3}$ nanowire at different growth times are shown in Fig. 6. The peak at $=4.25 \mathrm{eV}$ binding energy is thus mainly due to photoemission from $\mathrm{O} 2 p$ states. The peak at $=6.3 \mathrm{eV}$ is dominated by photoemission from $\mathrm{W} 5 d$ levels corresponded to a hybridized ' $\mathrm{W} 5 d-\mathrm{O} 2 p$ ' band. The $\mathrm{O} 2 p$ relatively disappeared or decreased in the valence band spectrum for samples (b) and (c) [33]. The onset of the valence band spectrum at $\sim 2.5 \mathrm{eV}$ could be assigned to the valence band maximum (VBM). The valence band maximum is almost purely due to the $\mathrm{O} 2 p$ states and the conduction band minimum is due to the W5d states.

There are $\mathrm{W}^{5+}$ and $\mathrm{W}^{4+}$ together with $\mathrm{W}^{6+}$ in sample (c), so that the residual W5d electrons appeared. Due to the empty conduction band of tungsten oxide associated with the W5 $d$ electrons [35], the peak near the Fermi level was attributed to the conduction band filled with the residual W5 $d$ electrons which is associated with the lower oxidation states. The intensity of the hybridized $\mathrm{W} 5 d-\mathrm{O} 2 p$ increased by the increase of the growth time.

\subsection{SEM analysis}

Figure $7 \mathrm{a}-\mathrm{d}$ show a series of SEM images at different growth times, depicting the morphology of the resulted tungsten oxide nanowires grown on the tungsten substrate. As can be seen in Fig. 7 a, by annealing at $800{ }^{\circ} \mathrm{C}$, with the oxidation of the tungsten substrate, a tungsten oxide thin film is created on the surface in the form of micro grains with cluster shapes. Grain boundaries act as nucleation sites for nanowire growth. The number of such activated nucleation sites is dependent to the annealing temperature. The nanowires on grains begin to germinate when the growth time increases to $5 \mathrm{~h}$, therefore, nanowire structures with small diameter could be formed and continue to grow as shown in Fig. 7b. As the growth time increases, the density of nanowires increases. Gradually, the growth in the
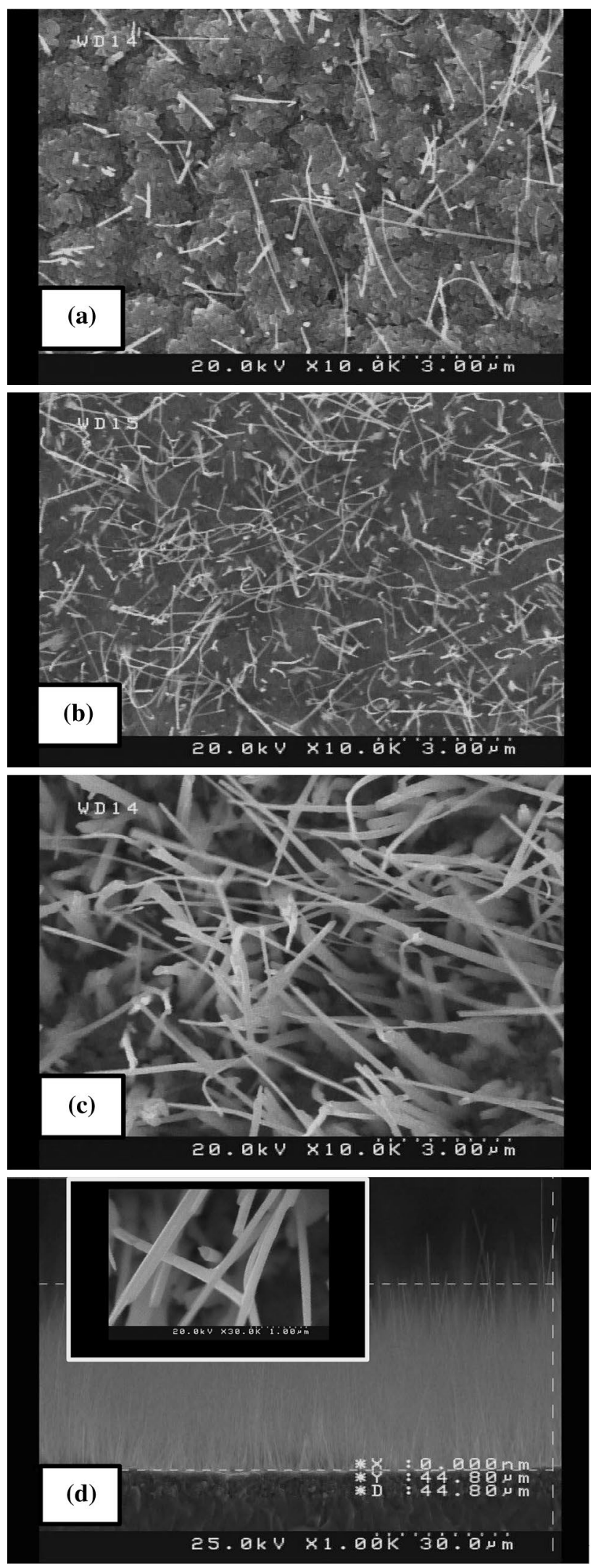

Fig. 7 Top view of SEM images of WO3 nanowire at different growth times: a 4, b 5 and $\mathbf{c} 6 \mathrm{~h}$. d Cross section of SEM images of WO3 nanowire for $6 \mathrm{~h}$ growth time 
axial direction becomes dominant compared to the lateral direction (Fig. 7c, d). In the inset of Fig. 7d, the image of nanowires has been shown in a smaller scale. The nanowire diameter were estimated in order of few tens of nanometers. It is clear that the surface of tungsten substrate are covered by thin layers of tungsten oxide nanowires, the length of nanowires increases with increasing the growth time. The average length of nanowires is $40 \mu \mathrm{m}$ after $5 \mathrm{~h}$. Moreover, with increasing growth time, it is noticed that the tungsten oxide nanowires grow preferentially along the axial direction. For the sample that grew up in a longer time much more nanowires are vertically distributed on the surfaces.

As shown in Fig. 7a-d, in the growth mechanism of tungsten oxide nanowires, at first the tungsten substrate was oxidized and tungsten oxide - a thin film - was created on the surface of tungsten substrate and then because of the thermal expansion coefficient between the thin film and the substrate at high annealing temperature, and based on atomic diffusion enhanced by compressive stress, tungsten oxide nanowires start to grow. In fact, by atomic diffusion at grain boundaries, to release the stress, tungsten oxide nanowires are grown on the surface of the tungsten oxide thin film $[26,27]$. It is known that the magnitude of this stress is proportional to the growth temperature, and there exists a threshold stress level and the corresponding temperature that initiates the growth of tungsten oxide nanowires; in this case, the threshold temperature to produce sufficient compressive stress for nanowire growth is $800{ }^{\circ} \mathrm{C}$, which according to previous studies is suitable for the growth of tungsten oxide nanowires [28, 29].

Fig. 8. shows the current as a function of voltage from 0 to $20 \mathrm{~V}$ for samples with KIETHLEY 2361 system. It could be observed that linear I-V curves are obtained and no indication of a hysteresis effect in any of the curves is observed, thus it is found that the current increases with voltage. By increasing the growth time, the conductivity of the sample increases, this may be due to an increase in the number of nanowires on the sample surface which increases the available free charge carriers. On the other hand, based on experimental results [30-32, 35], it was found that the number and size of nanowires have apparent influence on the electrical properties, which is in good agreement with the theoretical models [33, 34]. For studying the electrical conductivity in nanostructures, based on the Matthiessen's and Bloch-Grüneisen theory [34], the electrical resistivity depends on two factors: (1) the residual resistivity (due to structural scatterings caused by grain boundary, impurity and surface). (2) The temperature-dependent electrical resistivity (due to phonon scattering). It is noteworthy that in case of nanostructures; because the system size is smaller than the electron mean free path, the residual resistivity due to structural scatterings becomes important which limits the electron mean free path and decreases the electrical conductivities

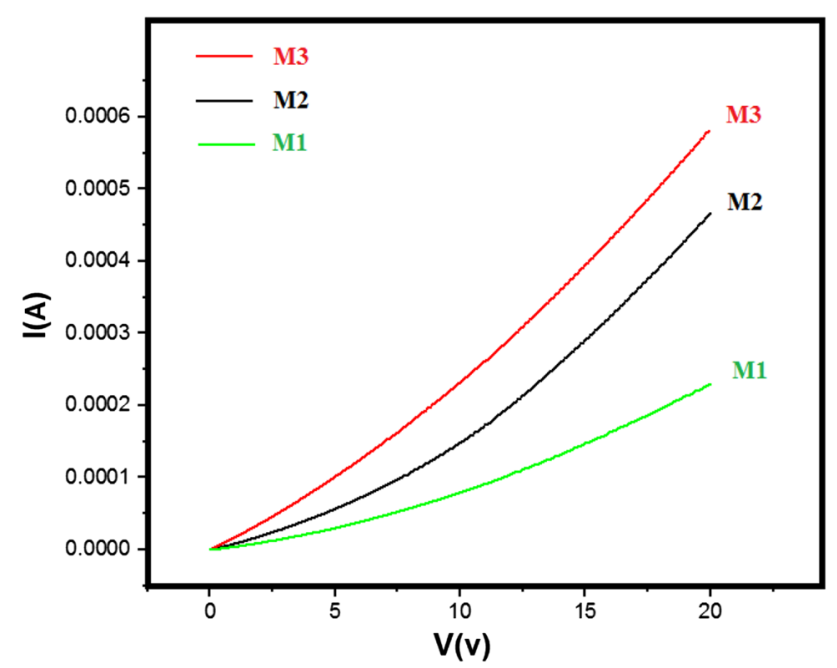

Fig. 8 Dependence of current with voltage of $\mathrm{WO}_{3}$ nanowire at different growth times: $\mathrm{M} 1=4, \mathrm{M} 2=5$ and $\mathrm{M} 3=6 \mathrm{~h}$

[35]. In this mechanism through electron-phonon scattering, the scattered electrons exchange energy with phonons so that phonons can transport through the grain boundary more easily than electrons.

\section{Conclusion}

The effect of growth time on the structural, morphological and electrical properties of $\mathrm{WO}_{3}$ nanowire has been observed. $\mathrm{WO}_{3}$ nanowires were grown directly on tungsten substrate in $800{ }^{\circ} \mathrm{C}$ using thermal evaporation method in a horizontal tube furnace in different growth times $(4,5$ and $6 \mathrm{~h}$ ). XRD pattern and XPS results, confirmed the cubic phase of the $\mathrm{WO}_{3}$ structure. Based on XRD results it is found that as growth time was increased, the crystallinity improved gradually and polycrystalline structure was forming. From the results, we could find that the crystallite size for dominant crystallographic (122) plane first decreased and then increases with increasing growth time while the dislocation density for dominant (122) plane has increased first and then decreased. In addition, the XPS results showed that the sample that grown at higher growth time exhibited two asymmetric $\mathrm{W} 4 d 5 / 2$ and $\mathrm{W} 4 d 3 / 2$ peak which can be assigned to $\mathrm{W} 5+$ and $\mathrm{W} 4+$ species, respectively, in the other words, the $\mathrm{W} 4 f$ core-level binding energy displays a strong dependence on the oxidation state of the $\mathrm{W}$ atom. In addition, at $6 \mathrm{~h}$ of growth time, the intensity of W $4 f 7 / 2$ of W $4 f$ core level varied and drastically increased. Surface morphology measured by FESEM revealed that with the oxidation of the tungsten substrate, the tungsten oxide thin film is created on the surface in the form of micro grains with cluster shapes and gradually the number and the length of tungsten oxide 
nanowires on the tungsten oxide grains increased when the growth time increased. Also, the change of the morphology due to changing the growth time can be highlighted and so that the growth in the axial direction becomes dominant compared to the lateral direction gradually. KIETHLEY instrument technique was used to investigate the electrical properties of tungsten oxide nanowires. This showed an increase in the average conductivity when the growth time was increased, this may be due to an increase in the number of nanowires on the sample surface which increases the number of available free charge carriers. Based on the results of this work, the control over the growth of tungsten oxide nanowire by adjusting the growth time creates effective insights for understanding the growth mechanism of tungsten oxide nanowires. This could contribute to the development of new nano-based electronic devices.

Open Access This article is distributed under the terms of the Creative Commons Attribution 4.0 International License (http://creativeco mmons.org/licenses/by/4.0/), which permits unrestricted use, distribution, and reproduction in any medium, provided you give appropriate credit to the original author(s) and the source, provide a link to the Creative Commons license, and indicate if changes were made.

\section{References}

1. H. Qiu, Y.F. Lu, Z.H. Mai, J. Appl. Phys. 91, 440 (2001)

2. Y. Koltypin, S.I. Nikitenko, A. Gedanken, J. Mater. Chem. 12, 1107 (2002)

3. C. Santato, M. Odziemkowski, M. Ulmann, J. Augustynski, J. Am. Chem. Soc. 123, 10639 (2001)

4. D. Dastan, Appl. Phys. A 123:699, 1-13 (2017)

5. D. Dastan, N. Chaure, M. Kartha, J. Mater. Sci. Mater. Electron. 28, 7784-7796 (2017)

6. C.G. Granqvist, Sol. Cells 60, 201 (2000)

7. K. Lee, W.S. Seo, J.T. Park, J. Am. Chem. Soc. 125, 3408 (2003)
8. C. Bigey, G. Maire, J. Catal. 196, 224 (2000)

9. J. Engweiler, J. harf, A. Baiker, J. Catal. 159, 259 (1996)

10. U.O. Turyan, B. Krasovec, T. Orel, R. Saraidorov, D. Reisfeld, Mandler, Adv. Mater. 12, 330 (2000)

11. W.M. Qu, W. Wlodarski, Sens. Actuators B 64, 42 (2000)

12. K.H. Lee, Y.K. Fang, W.J. Lee, J.J. Ho, K.H. Chen, K.S. Liao, Sens. Actuators B 69, 96 (2000)

13. Z. Liu, Y. Bando, C. Tang, Chem. Phys. Lett. 372, 179 (2003)

14. C. Klinke, J.B. Hannon, L. Gignac, K. Reuter, P. Avouris, J. Phys. Chem. B 38, 17787 (2005)

15. W. Yu, C. Pan, Mater. Chem. Phy. 115, 74 (2009)

16. G. Gu, B. Zheng, W.Q. Han, S. Roth, J. Liu, Nano Lett. 8, 849 (2002)

17. H.S. Shim, J.W. Kim, Y.E. Sung, W.B. Kim, Solar Energy Mater. Solar Cells 93, 2062 (2009)

18. Y. Li, Y. Bando, D. Golberg, Adv. Mater. 15, 1294 (2003)

19. G. Gu, B. Zheng, W.Q. Han, S. Roth, J. Liu, Nano Lett. 2, 849 (2002)

20. P.M. Rao, I.S. Cho, X. Zheng, Proc. Combust. Inst. 34, 2187-2195 (2013)

21. S.S. Kalanur, Y.J. Hwang, S.Y. Chae, O.S. Joo, J. Mater. Chem. A 1, 3479-3488 (2013)

22. X. Liu, F. Wang, Q. Wang, Phys. Chem. Chem. Phys. 14, 7894 $7911(2012)$

23. S.K. Biswas, J. Baeg, S. Moon, K. Kong, W. So, J. Nanopart. Res. 14, 1-12 (2012)

24. J. Su, X. Feng, J.D. Sloppy, L. Guo, C.A. Grimes, Nano Lett. 11, 203-208 (2011)

25. H. Kim, K. Senthil, K. Yong, Mater. Chem. Phys. 120, 452-455 (2010)

26. W. Shim et al., Nano Lett. 9, 18 (2009)

27. J. Ham et al., Nano Lett. 9, 2867-2872 (2009)

28. K. Nagato et al., Appl. Phys. Express 1, 014005 (2008)

29. Y. Kojima, K. Kasuya, K. Nagato, T. Hamaguchi, M. Nakao, J. Vac. Sci. Technol. B 26, 1942 (2008)

30. F. Volklein et al., Nanotechnology 20, 32 (2009)

31. N. Stojanovic et al., Appl. Phys. Lett. 95, 9 (2009)

32. S. Yoneoka et al., Nano Lett. 12, 683 (2012)

33. W.G. Ma, X. Zhang, Int. J. Heat Mass Transf. 58, 639 (2013)

34. R.A. Matula, J. Phys. Chem. Ref. Data 8, 1147 (1979)

35. D. Dastan, S.W. Gosavi, N.B. Chaure, Macromol. Symp. 347, 81-86 (2015) 